\title{
Physiological response of Pichia pastoris GS115 to methanol-induced high level production of the Hepatitis B surface antigen: catabolic adaptation, stress responses, and autophagic processes
}

Ana Leticia Vanz ${ }^{1}$, Heinrich Lünsdorf², Ahmad Adnann ${ }^{2,3}$, Manfred Nimtz², Chandrasekhar Gurramkonda 2,4, Navin Khanna ${ }^{4}$ and Ursula Rinas ${ }^{1,2^{*}}$

\begin{abstract}
Background: Pichia pastoris is an established eukaryotic host for the production of recombinant proteins. Most often, protein production is under the control of the strong methanol-inducible aox 1 promoter. However, detailed information about the physiological alterations in P. pastoris accompanying the shift from growth on glycerol to methanol-induced protein production under industrial relevant conditions is missing. Here, we provide an analysis of the physiological response of $P$. pastoris GS115 to methanol-induced high-level production of the Hepatitis B virus surface antigen (HBsAg). High product titers and the retention of the protein in the endoplasmic reticulum (ER) are supposedly of major impact on the host physiology. For a more detailed understanding of the cellular response to methanol-induced HBsAg production, the time-dependent changes in the yeast proteome and ultrastructural cell morphology were analyzed during the production process.
\end{abstract}

Results: The shift from growth on glycerol to growth and HBsAg production on methanol was accompanied by a drastic change in the yeast proteome. In particular, enzymes from the methanol dissimilation pathway started to dominate the proteome while enzymes from the methanol assimilation pathway, e.g. the transketolase DAS1, increased only moderately. The majority of methanol was metabolized via the energy generating dissimilatory pathway leading to a corresponding increase in mitochondrial size and numbers. The methanol-metabolism related generation of reactive oxygen species induced a pronounced oxidative stress response (e.g. strong increase of the peroxiredoxin PMP20). Moreover, the accumulation of HBsAg in the ER resulted in the induction of the unfolded protein response (e.g. strong increase of the ER-resident disulfide isomerase, PDI) and the ER associated degradation (ERAD) pathway (e.g. increase of two cytosolic chaperones and members of the AAA ATPase superfamily) indicating that potential degradation of HBsAg could proceed via the ERAD pathway and through the proteasome. However, the amount of HBsAg did not show any significant decline during the cultivation revealing its general protection from proteolytic degradation. During the methanol fed-batch phase, induction of vacuolar proteases (e.g. strong increase of APR1) and constitutive autophagic processes were observed. Vacuolar enclosures were mainly found around peroxisomes and not close to HBsAg deposits and, thus, were most likely provoked by peroxisomal components damaged by reactive oxygen species generated by methanol oxidation.

(Continued on next page)

\footnotetext{
* Correspondence: ursula.rinas@helmholtz-hzi.de

${ }^{1}$ Technical Chemistry - Life Science, Leibniz University of Hannover,

Hannover, Germany

${ }^{2}$ Helmholtz Centre for Infection Research, Inhoffenstrasse 7, Braunschweig

38124, Germany

Full list of author information is available at the end of the article
} 
(Continued from previous page)

Conclusions: In the methanol fed-batch phase $P$. pastoris is exposed to dual stress; stress resulting from methanol degradation and stress resulting from the production of the recombinant protein leading to the induction of oxidative stress and unfolded protein response pathways, respectively. Finally, the modest increase of methanol assimilatory enzymes compared to the strong increase of methanol dissimilatory enzymes suggests here a potential to increase methanol incorporation into biomass/product through metabolic enhancement of the methanol assimilatory pathway.

Keywords: Pichia pastoris, Proteome, Aox1 promoter, Carbon metabolism, ER stress, Autophagy

\section{Background}

The methylotrophic yeast Pichia pastoris is nowadays a well-established eukaryotic host for the heterologous production of technical enzymes and also for protein pharmaceuticals $[1,2]$. P. pastoris naturally possesses two different genes (aox1 and aox2) encoding alcohol oxidases for oxidation of methanol, the first step in methanol degradation [3]. Aox1 is highly expressed during growth on methanol, thus the strong aox 1 promoter is utilized most often to drive the production of foreign proteins. Another attempt to enhance the yield of the target protein is to increase its chromosomal gene dosage which - up to a certain target-protein dependent extent - can further increase product levels [4-7]. However, increasing the gene copy number beyond a target protein-dependent threshold can have a severe impact on cell growth and viability including a reduced level of the target protein [5,8-10]. In particular, overburdening the cellular protein export machinery for production of extracellular proteins can cause severe stress responses [11]. The passage of export-destined proteins through the secretory pathway often presents a bottleneck leading in many cases to partial retention of the target protein in the endoplasmic reticulum (ER) and a transcriptional upregulation of the Unfolded Protein Response (UPR) and the ER-Associated Degradation (ERAD) pathway [12,13].

In this study we investigated the physiological changes accompanying the methanol-induced high-level production of HBsAg, the major surface antigen of the Hepatitis $B$ virus (HBV) employed for vaccination against $\mathrm{HBV}$, in $P$. pastoris under industrially relevant conditions. The antigen is a very stable and also a very hydrophobic protein and not secretable in yeast expression systems [14]. Thus, production occurs as intracellular protein in $P$. pastoris GS115 carrying 8 copies of the gene encoding mature HBsAg without employing yeastderived secretory signals [4]. Cells are grown on defined medium in a controlled fed-batch procedure maintaining the methanol concentration at $6 \mathrm{~g} \mathrm{~L}^{-1}$ through continuous methanol feeding during the production phase [15]. Although not destined for export the HBsAg is translocated into the ER where it is retained leading to a bulging of the ER into cloud-shaped irregular formations
[14]. The very hydrophobic HBsAg is not further passaged in the secretory pathway but assembles into well-ordered multi-layered lamellar structures in the ER which are transformable into virus-like particles (VLPs) during down-stream processing [14]. The highlevel production and the retention of the protein in the ER are supposedly of major impact on the cell physiology. For a more detailed understanding of the cellular response to methanol-induced high level HBsAg production, we followed the changes in the yeast proteome and the ultrastructural cell morphology during the HBsAg production process.

\section{Results and discussion}

The physiological alterations accompanying the adaption of recombinant Pichia pastoris to methanol-induced high-level production of HBsAg were followed by proteome analysis and electron microscopy. Cells were grown initially on glycerol, and after depletion of glycerol, methanol was added to a final concentration of $6 \mathrm{~g} \mathrm{~L}^{-1}$ to initiate HBsAg production. This methanol concentration was kept constant through appropriate methanol feeding for the remaining fed-batch phase of the cultivation [15]. After an initial growth arrest and a short period of adaptation to methanol ( 3-4 h), cells started to consume methanol and the biomass increased from 60 to $100 \mathrm{~g} \mathrm{~L}^{-1}$ cell dry mass during the first $90-100 \mathrm{~h}$ of the methanol feeding phase [15]. During this period, the intracellular $\mathrm{HBsAg}$ levels increased strongly reaching a final maximum of $6-7 \mathrm{~g} \mathrm{~L}^{-1}$ (thereof $30 \%$ soluble $\mathrm{HBsAg}$ competent for assembly into VLPs). Beyond this period, cell viability declined concomitant with a decrease of the soluble fraction of HBsAg [15]. Thus, this production process is characterized by four distinct phases: $i$. glycerol batch phase, ii. adaptation phase to methanol, iii. production phase, and $i v$. decline phase. From all these phases representative samples were taken for proteome analysis. The complete list of all identified proteins with their corresponding changes and classified into functional categories and a 2D gel image indicating all identified proteins are found in the Additional file 1 . Moreover, cell physiological changes were also followed by ultrastructural analysis using transmission electron microscopy. 


\section{Carbon metabolism: cell adaptation to carbon source change from glycerol to methanol}

The intracellular proteome of Pichia pastoris exhibited a dramatic change in response to the shift from growth on glycerol to production and growth on methanol (Figure $1 \mathrm{~A}$ and $\mathrm{B}$ ).

\section{Methanol metabolism}

In particular, proteins required for methanol utilization which were only present in minor amounts during growth on glycerol started to dominate the proteome after the onset of the methanol feeding phase. Surprisingly, a major protein which accumulated during growth on methanol was identified as alcohol oxidase 1 (AOX1) (Figures 1A-C, 2 and Additional file 2). This HBsAg producing strain was first classified as $\mathrm{Mut}^{\mathrm{s}}$ [4], hence the presence of aox1 and the accumulation of AOX1 unexpected. A comprehensive analysis verified the formation of AOX1 as well as the presence of the intact encoding gene aox1 (for details please refer to Additional file 2).
(A)

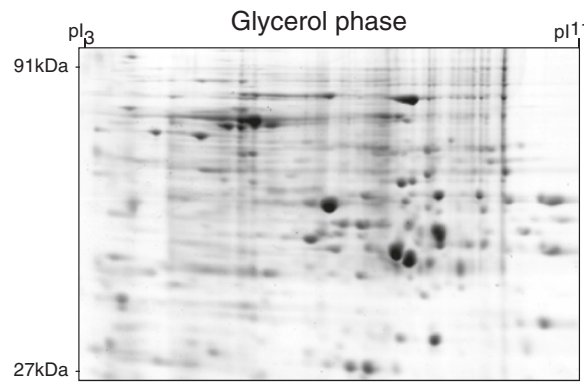

(B)

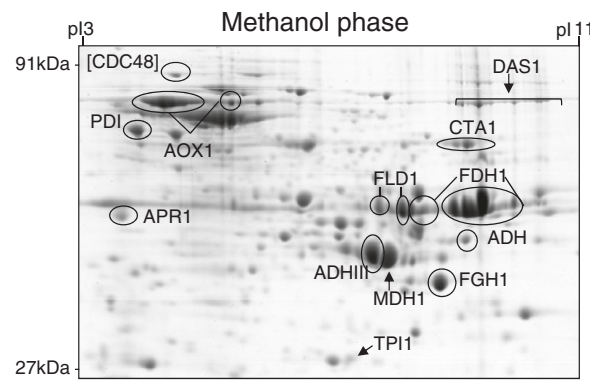

(C) Methanol pathway

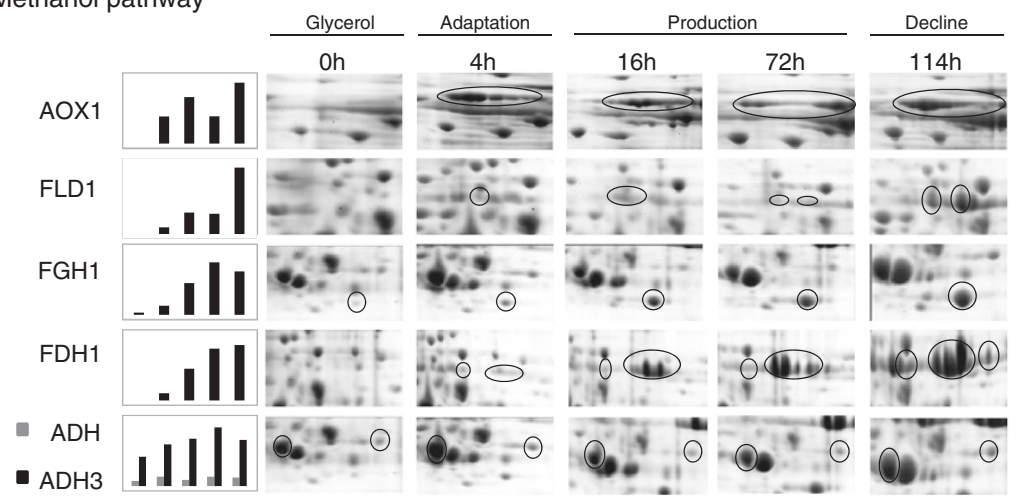

(D) Oxidative stress

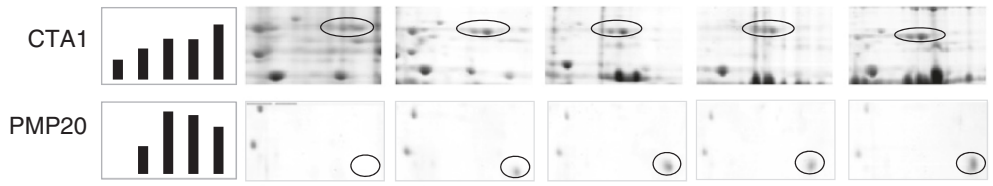

(E) ER stress response and degradation

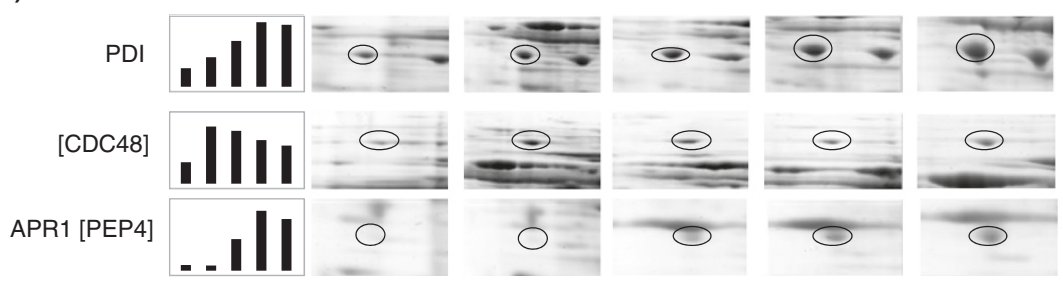

Figure 1 Change of the intracellular proteome of $P$. pastoris GS115 in response to methanol-induced high-level production of the Hepatitis B surface antigen. Sections of 2D gels representing parts of the intracellular proteome $(\mathbf{A})$ at the end of the glycerol batch phase and (B) 114 hours after the onset of methanol feeding. For encircled protein spots (dark lines) time-dependent changes are given below. Magnified 2D gel sections from samples taken at the end of the glycerol batch phase and 4, 16, 72 and 114 hours after the onset of methanol feeding with representative proteins involved in (C) methanol metabolism and (D) oxidative and (E) ER stress responses (including also vacuolar degradation). 


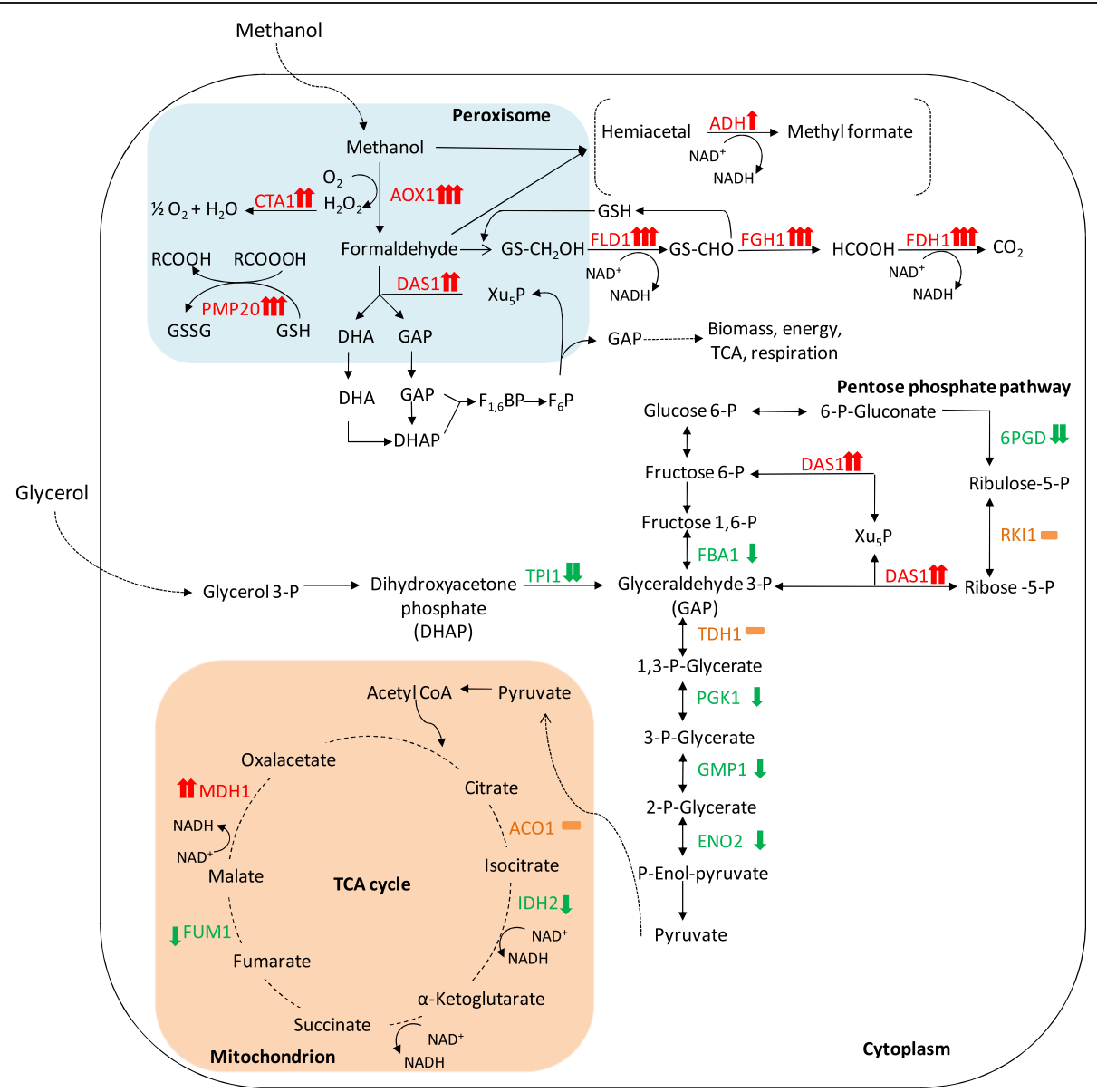

Figure 2 Simplified carbon metabolic network. Identified enzymes from pathways involved in glycerol and methanol metabolism, from the glycolytic and pentose phosphate pathway, and TCA cycle and their changes in response to the shift from growth on glycerol to growth and HBsAg production on methanol. The red arrow $(\uparrow)$ indicates an increasing and the green arrow $(\downarrow)$ a decreasing amount of the enzyme in the methanol fed-batch phase. An orange dash (-) indicates no significant change. One arrow indicates small (0.6-1 log2 change), two arrows strong (1-4 log2 change) and three arrows very strong changes (> 4 log2 change). Abbreviations (enzymes): AOX1, alcohol oxidase 1; CTA1, catalase; PMP20, peroxiredoxin; FLD1, formaldehyde dehydrogenase; FGH1, S-formylglutathione hydrolase; FDH1, NAD(+)-dependent formate dehydrogenase; ADH, alcohol dehydrogenase; DAS1, transketolase (dihydroxyacetone synthase); TPI1, triose phosphate isomerase; FBA1, fructose 1,6-bisphosphate aldolase; TDH1, glyceraldehyde-3-phosphate dehydrogenase; PGK1, 3-phosphoglycerate kinase; GMP1, tetrameric phosphoglycerate mutase; ENO2, enolase; 6PGD, 6-phosphogluconate dehydrogenase; RKI1, 1-ribose-5-phosphate ketol-isomerase; ACO1, aconitase; IDH2, subunit of mitochondrial $N A D(+)$-dependent isocitrate dehydrogenase; FUM1, fumarase; MDH1, mitochondrial malate dehydrogenase.

Thus, high level recombinant protein production may lead to impaired growth on methanol despite the presence of functional aox1.

In addition to AOX1 also the $\mathrm{NAD}^{+}$-dependent formate dehydrogenase (FDH1), the enzyme catalyzing the last step in the methanol dissimilation pathway increased to very high levels in the methanol feeding phase (Figures 1A-C and 2). The other two enzymes of the methanol dissimilation pathway, the S-(hydroxymethyl)-glutathione dehydrogenase (FLD1) and S-formylglutathione hydrolase (FGH1), also increased strongly but not as pronounced as AOX1 and FDH1 (Figures 1A-C and 2).

Interestingly, AOX1 already accumulated to high levels during the adapation phase to methanol (Phase II), while the subsequent enzymes of the methanol dissimilation pathway reached their highest concentrations in the production phase (Phase III) or even later in the decline phase (Phase IV) (Figure 1A-C). Methanol is not only oxidized by Pichia pastoris in the dissimilation pathway for potential energy generation through reoxidation of NADH in the respiratory chain but can also be incorporated into biomass in the assimilation pathway. In this case, formaldehyde, which is formed through oxidation of methanol by AOX1 (or AOX2), is not further converted to carbon dioxide, but condensed with xylulose 5-phosphate (Xu5P), through the action of dihydroxyacetone synthase (DAS1), a special transketolase which converts formaldehyde and Xu5P into the central 
C3-compounds dihydroxyacetone (DHA) and glyceraldehyde 3-phosphate (GAP) [16] (Figure 2). In contrast to the strong increase of the enzymes from the methanol dissimilation pathway, the level of DAS1 increased only moderately during the production phase (Figures 1A, B and 2) suggesting that the majority of methanol is processed through the dissimilation pathway leading to the generation of NADH and carbon dioxide. In fact, a carbon mass balance analysis revealed that $70-80 \%$ of the methanol metabolized is converted into carbon dioxide in the methanol fed-batch phase (data not shown). Interestingly, the mitochondrial area within cellular cross sections increased significantly during the methanol fedbatch phase (Figure 3) probably as a result of the elevated NADH supply from methanol dissimilation and the enhanced energy demand for growth, production and cell maintenance (e.g. repair and recycling).

\section{Glycolysis, pentose phosphate pathway, and tricarboxylic acid (TCA) cycle}

In contrast to the strong increase of the enzymes from the methanol utilizing pathways, in particular enzymes from the methanol dissimilation pathway, the enzymes from the glycolytic and the pentose phosphate pathway either decreased during the methanol fed-batch phase or did not show significant changes (Figure 2). A more pronounced decrease was observed for the triose phosphate isomerase (TPI1), the glycolytic enzyme required for channeling glycerol into the central carbon metabolic pathways (Figures 1A, B and 2 and Additional file 1). The enzymes of the TCA cycle did not show significant changes except for the mitochondrial malate dehydrogenase $(\mathrm{MDH} 1)$ which increased strongly during the methanol feeding phase (Figures 1A, B and 2 and Additional file 1). MDH1 was already a prominent component of the intracellular proteome during growth on glycerol but increased even further in the methanol feeding phase (Figure 1A and B). Interestingly, the corresponding homolog in $A$. niger was also identified as major part of the intracellular proteome in well-aerated bioreactor cultures [17].

\section{Ethanol metabolism}

$P$. pastoris is generally classified as Crabtree-negative yeast and glycerol and methanol are not considered as fermentable carbon sources. However, two alcohol dehydrogenases (ADH and mitochondrial ADHIII) were identified in the proteome during growth on glycerol with ADHIII as a prominent component of the intracellular proteome (Figure 1A and C). The presence of alcohol dehydrogenases indicated the formation of ethanol in the glycerol batch phase which was confirmed by gas chromatography $\left(1.3 \mathrm{~g} \mathrm{~L}^{-1}\right.$ ethanol at the end of the glycerol batch phase). Previous reports also documented ethanol formation by $P$. pastoris during growth on excess glycerol $[18,19]$. Surprisingly, the amount of both enzymes increased even further in the methanol fedbatch phase (Figure 1A-C; ethanol concentration: $0.3 \mathrm{~g} \mathrm{~L}^{-1}$ after 112 hours of growth on methanol) indicating a potential involvement in metabolic activities beyond ethanol metabolism. In fact, it has been suggested that proteins of the $\mathrm{ADH}$ family from other methylotrophic yeasts (e.g. C. boidinii) are involved in fomaldehyde detoxification through formation of methyl formate [20]. A similar role may be also attributable to ADHs in P. pastoris.

\section{Stress responses}

In the methanol fed-batch phase the cells are exposed to dual stress; stress resulting from methanol degradation and stress resulting from the production of the recombinant protein. The first, AOX catalyzed step during methanol utilization generates formaldehyde and hydrogen peroxide, both toxic compounds [16]. The delayed
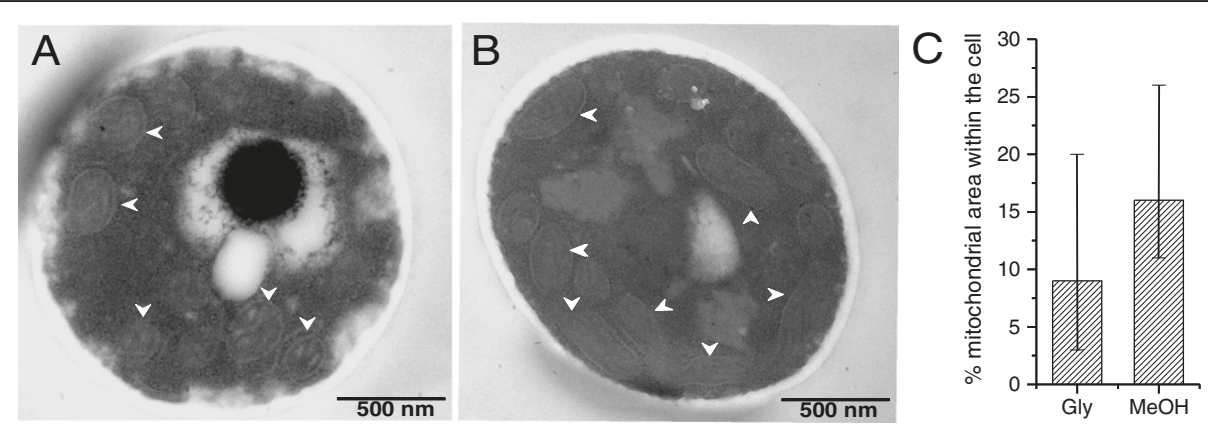

Figure 3 Mitochondrial morphology and size changes during methanol-induced high-level production of the Hepatitis B surface antigen. Transmission electron micrographs of P. pastoris GS115 (A) at the end of the glycerol batch phase and (B) 112 hours after the onset of methanol feeding. White arrow heads indicate mitochondria. (C) Quantification of the average total mitochondrial area within the cross sectional average cell area. The vertical bars encompass individual cell variance, e.g. the lowest and highest total mitochondrial area observed in a single cell in each growth phase; (left column) at the end of the glycerol batch phase and (right column) 112 hours after the onset of methanol feeding. 
accumulation of formaldehyde processing enzymes compared to the rapid accumulation of AOX after the onset of methanol feeding (cf. preceding paragraph and Figure $1 \mathrm{~A}-\mathrm{C}$ ) suggests a considerable negative impact of formaldehyde on the cells, in particular in the beginning of the methanol fed-batch phase.

\section{Oxidative stress response}

Methanol metabolism is mainly localized in the peroxisomes, membrane surrounded organelles which harbor the enzymes required for the initial steps of methanol metabolism (e.g. AOX, DAS1). During oxidation of methanol reactive oxygen species such as hydrogen peroxide but also peroxidated molecules are generated which need to be removed to prevent or minimize corresponding cell damage. At least two peroxisomal enzymes which are involved in the removal of reactive oxygen species, catalase (CTA1), which removes hydrogen peroxide $[16,21,22]$, and a glutathione peroxidase or peroxiredoxin (PMP20), which removes peroxidated molecules, e.g. lipid hydroperoxides [16,22,23], increased strongly in the methanol fed-batch phase (Figures 1A, B, D and 2). CTA1 was already present in significant amounts during growth on glycerol and increased further in the adaptation and production phases (Figures 1A, B, D and 2). In contrast to CTA1, PMP20 was virtually absent during growth on glycerol but increased immediately in the methanol adaptation phase (Figures 1D and 2) indicating a more important role in methanol-related detoxification of reactive oxygen species. In fact, deletion of $p m p 20$ was more deleterious than deletion of ctal in methylotrophic yeast knockout strains (C. boidinii and $P$. pastoris) exposed to methanol $[23,24]$. It has been suggested that the presence of PMP20 is essential for maintaining peroxisomal membrane integrity during growth on methanol through removal of oxidized lipids [25]. Cyclophilin B, a cytoplasmic peptidylprolyl cis-trans isomerase (Cpr1), reported as environmental (oxidative)-stress responsive protein in Saccharomyces cerevisiae [26], also increased immediately in the methanol adaptation phase (Additional file 1) suggesting a stressresponsive function also for $P$. pastoris.

\section{Induction of UPR and ERAD pathway}

HBsAg is a very stable and also a very hydrophobic protein able to form VLPs during downstream processing [15]. The VLPs are further stabilized by intra- and intermolecular disulfide bonds [27]. During its methanolinduced high-level production $\mathrm{HBsAg}$ is translocated into the ER but not further processed in the secretory pathway [14]. The accumulation of HBsAg in the ER is leading to an expansion of the ER which bulges into cloud-shaped irregular formations. Thus, an induction of the UPR and a corresponding increase of ER resident chaperones or foldases would not be surprising. In fact, the amount of the UPR-inducible and ER-resident disulfide isomerase (PDI) increased strongly in the methanol fed-batch phase (Figure 1A, B, and E). Moreover, other UPR-induced proteins such as the mitochondrial chaperone SSC1 [28] also increased significantly during HBsAg production (Additional file 1). In addition, two cytosolic chaperones and members of the AAA ATPase superfamily $(\mathrm{ClpB}=\mathrm{hsp} 104$ and the AAA ATPase PAS_FragD_0026=Cdc48) also revealed a strong increase in the methanol fed-batch phase (Figure 1A, B, E and Additional file 1) indicating an activation of the ERAD pathway. In $S$. cerevisiae, the homologs of both proteins have been identified as ER-stress responsive proteins, which participate in energy-driven disaggregation and degradation of ERAD substrates [29-31]. Hsp104 cooperates with hsp70 and hsp40 in disassembling protein aggregates for either proper refolding or degradation [29,31,32]. The AAA ATPase Cdc48 operates on the cytosolic part of the ER membrane actively involved in dragging misfolded proteins from the ER for subsequent degradation by the proteasome [33-35]. Here, their increase in response to ER stress was also verified for $P$. pastoris.

\section{Induction of other degradation pathways and autophagic processes}

Moreover, a strong increase of the vacuolar aspartyl protease APR1 (PEP4 in S. cerevisiae) was observed in the methanol fed-batch phase (Figure 1A, B, E and Additional file 1) suggesting the induction of vacuolar degradation pathways in addition to ERAD. An electron microscopic examination of the cells revealed a drastic change in vacuole morphology after the start of the methanol fed-batch phase (Figure 4). At the end of the glycerol batch phase, the majority of cells possessed large spherical vacuoles of which many contained autophagic bodies. The appearance of spherical vacuoles with autophagic bodies indicate the onset of nutrient limiting conditions and the accompanying recycling of cell material [36]. After the start of methanol feeding, the number of cells with spherical vacuoles declined strongly and, instead, cells with irregularly shaped vacuoles increased in number (Figure 4). A closer electron microscopic examination revealed invagination of vacuoles (Figure 5) as is typically observed during peroxisome degradation by microautophagy (micropexophagy) $[37,38]$. Micropexophagy requires high levels of ATP [39] most likely available in the methanol fed-batch phase through primarily dissimilatory methanol catabolism. Interestingly, vacuolar enclosure was mainly related to peroxisomes (for details see Figure 5) and not to HBsAg deposits suggesting that vacuolar degradation pathways were not induced by $\mathrm{HBsAg}$ accumulation but most likely by damaged peroxisomes. In other cases, 

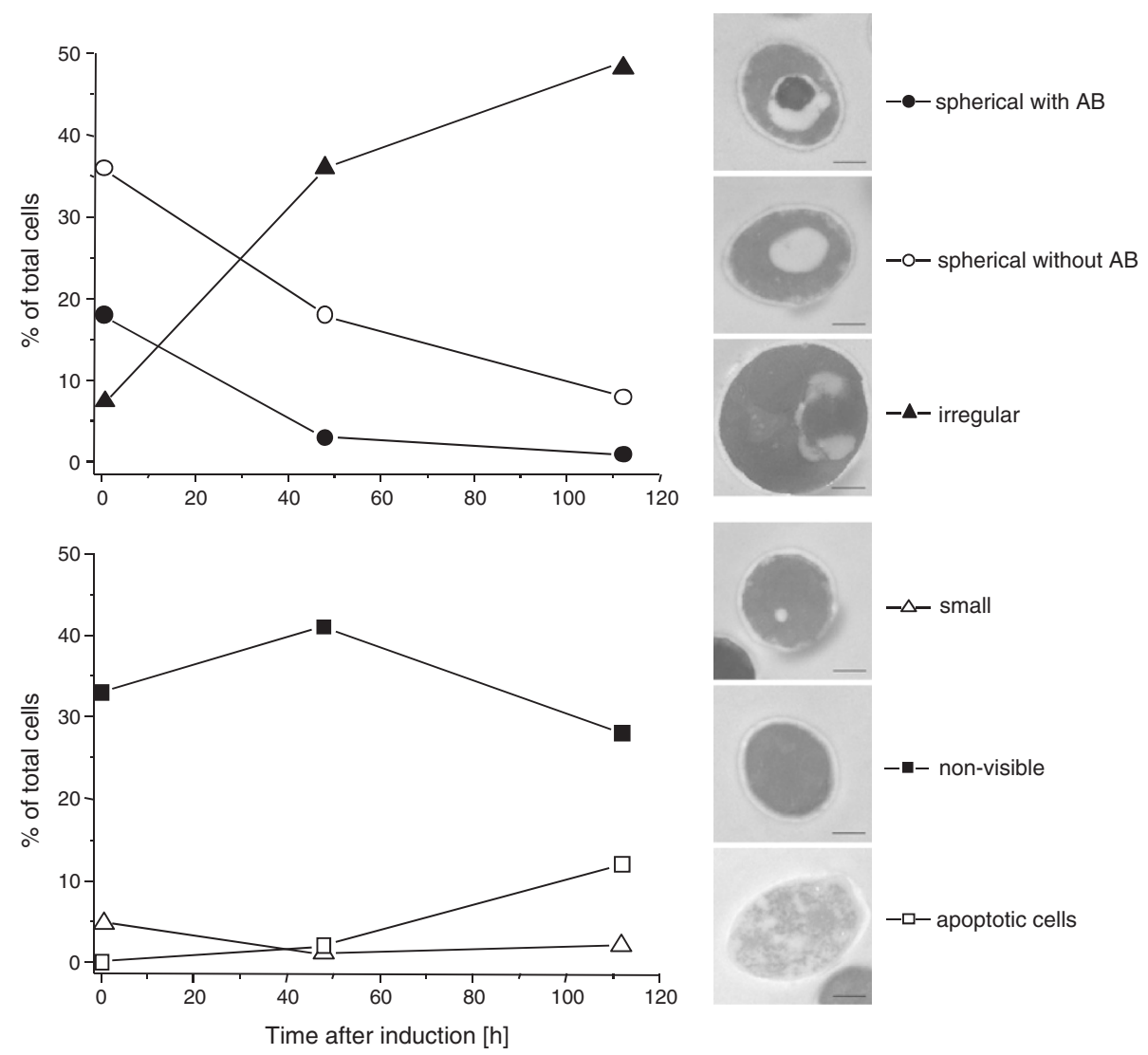

Figure 4 Vacuole morphology changes in P. pastoris GS115 during methanol-induced high-level production of HBsAg. Time-dependent change in the percentage of cells containing large spherical vacuoles with autophagic bodies (AB, $\bullet$ ), cells containing large spherical vacuole without autophagic bodies $(\mathrm{O})$, cells containing irregular vacuoles $(\boldsymbol{\Lambda})$, cells with small vacuoles $(\boldsymbol{\Delta})$, cells without any visible vacuoles $(\mathbf{\square})$, and apoptotic cells $(\square)$. The bar in the electron micrographs of representative cells corresponds to $500 \mathrm{~nm}$.

activation of autophagic processes have been reported in S. cerevisiae and mammalian cells upon induction of ER stress through addition of reducing agents [40] and tunicamycin and thapsigargin [41], respectively. Moreover, the analysis of the interactome of a degradation-prone and secreted Fab fragment in P. pastoris revealed mainly proteasomal degradation but also degradation via vacuolar pathways [42]. Also, analysis of the effects of producing folded-state stability variants of human lysozyme on the activation of stress responsive pathways revealed a reverse correlation of protein stability versus activation of degradative processes such as ERAD and ER-phagy, apparent through enhanced expression of e.g. sec61 and e.g. atg1, respectively [12]. However, in our case invaginated vacuoles were either closely connected to peroxisomes (Figure 5) or otherwise did not show any clear connection to other organelles (e.g. mitochondria or ER). Autophagy of peroxisomes (pexophagy) has been previously reported for $P$. pastoris upon shifting from methanol to ethanol or glucose but not during growth on methanol. Our findings suggest that constitutive autophagic recycling of peroxisomes might be part of the house-keeping machinery of Pichia pastoris also under methanol growth conditions helping cells to deal with damage caused by reactive oxygen species created through methanol oxidation. Vacuolar enclosure of peroxisomes was already apparent in the middle of the production phase (Figures 4 and 5D) and increased further during the ongoing methanol fed-batch phase (Figures 4 and 5). Indeed, it has been shown for the methylotrophic yeast Hansenula polymorpha that constitutive pexophagy is vital during growth on methanol as mutant cells with defects in autophagy displayed reduced vitality [43] and damaged peroxisomes are rapidly subjected to autophagic degradation in $H$. polymorpha [44]. Thus, the strong increase in the vacuolar protease APR1 during the methanol fed-batch phase might not be related to HBsAg production but to vacuolar degradation of damaged peroxisomes.

\section{Conclusions}

The shift from growth on glycerol to growth and production on methanol leads to a drastic change in the yeast proteome. In particular, enzymes from the 

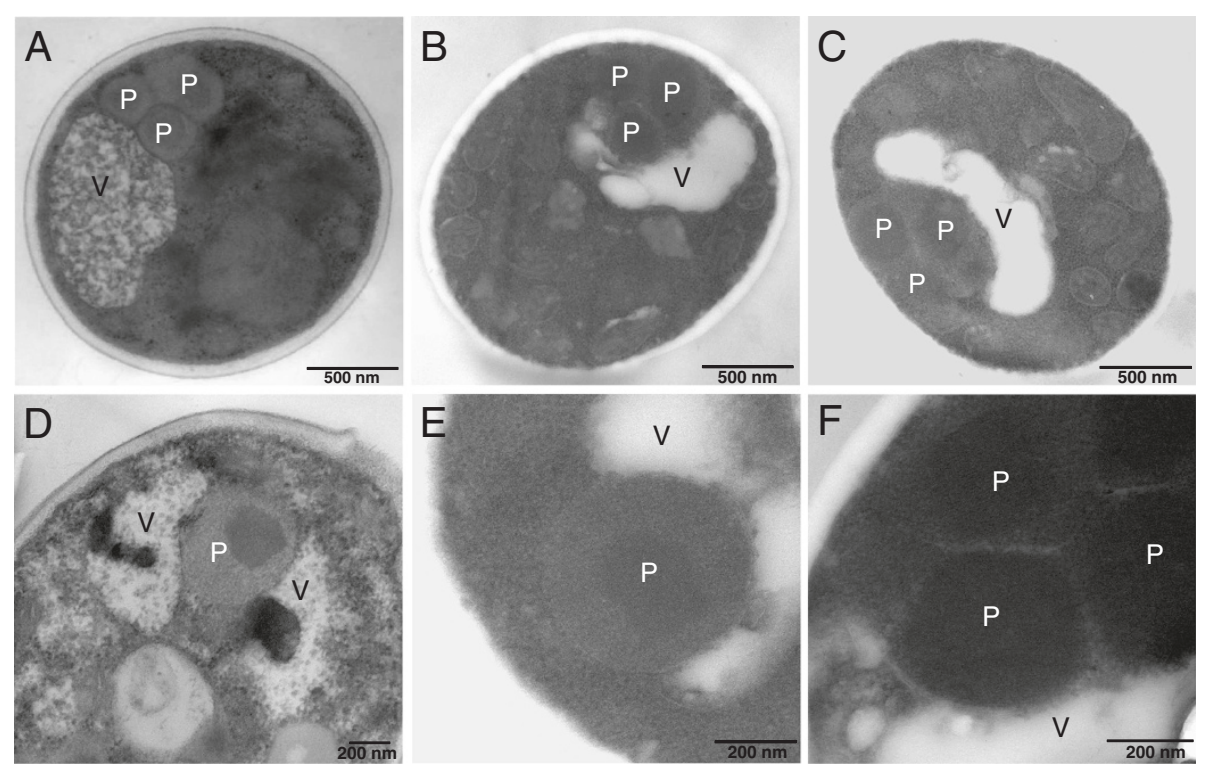

Figure 5 Peroxisome sequestration via micropexophagy during methanol-induced high-level production of HBsAg. (A-C) Representative transmission electron micrographs of ultrathin sectioned cells of $P$. pastoris GS115 growing for 112 hours on methanol. (D-F) Close-up views of vacuoles sequestering peroxisomes in cells growing for (D) 48 and (E, F) 112 hours on methanol. Abreviations: V, vacuole; P, peroxisome.

methanol dissimilation pathway start to dominate the proteome while enzymes from the methanol assimilation pathway, e.g. the transketolase DAS1, only show a moderate increase during the production phase suggesting this pathway as a potential target for metabolic engineering to enhance methanol assimilation, e.g. though enhanced expression of genes from the methanol assimilatory pathway [e.g. 45]. Moreover, the strong increase of alcohol dehydrogenases, ADHs, in the methanol-fed batch phase indicate that ADHs may play an important role in formaldehyde detoxification in P. pastoris.

The accumulation of HBsAg in the ER of P. pastoris leads to the induction of the UPR and the ERAD pathway suggesting that potential degradation of HBsAg may proceed via the ERAD pathway and through the proteasome. On the other hand, there is no significant decrease in the concentration of HBsAg even after prolonged cultivation [15] suggesting that the (lamellar) HBsAg deposits in the ER [14] are well protected from proteolytic degradation. Electron microscopic investigations revealed autophagic processes mainly related to peroxisome degradation and not to degradation of HBsAg deposits. Thus, the activation of autophagic processes also by ER-derived misfolded proteins as reported for mammalian [41] but also for fungal systems [46] might depend on specific protein properties and also on environmental conditions. During methanol-induced production of HBsAg in P. pastoris, autophagic processes are clearly related to peroxisome turnover most likely provoked by peroxisomal proteins and components damaged by reactive oxygen species.

\section{Methods}

\section{Strain and growth conditions}

The construction of the P. pastoris strain GS115 carrying 8-copies of the HBsAg structural gene under the control of the aox 1 promoter and displaying a $\mathrm{Mut}^{\mathrm{S}}$ phenotype was described before [4]. Cells were grown on defined medium in a fed-batch procedure as described earlier [15]. High-level production of HBsAg was started after batch growth on glycerol through the addition of methanol to a final concentration of $6 \mathrm{~g} \mathrm{~L}^{-1}$. This methanol concentration was kept constant by continuous methanol feeding throughout the entire production phase [15].

\section{Sample preparation}

Cells were harvested by centrifugation and washed with phosphate buffered saline to remove extracellular proteins and other contaminants. The remaining pellet was resuspended in extraction buffer $\left(20 \mathrm{mmol} \mathrm{L}^{-1}\right.$ Tris$\mathrm{HCl}$, pH 7.6, $10 \mathrm{mmol} \mathrm{L}^{-1} \mathrm{NaCl}, 0.5 \mathrm{mmol} \mathrm{L}^{-1}$ deoxycholate, $1 \mu \mathrm{g} \mathrm{mL} \mathrm{m}^{-1}$ pepstatin). Cell disruption was accomplished by grinding in liquid nitrogen using a Mortar Grinder (RM 100, Retsch Gmbh \&Co. KG, Germany). The cell debris was removed by centrifugation $\left(22000 \times \mathrm{g}\right.$, at $4^{\circ} \mathrm{C}$ for $\left.30 \mathrm{~min}\right)$ and $1 \mathrm{~mL}$ of the subsequent supernatant treated for 15 min with $7 \mu \mathrm{L}$ of nuclease mix $\left(0.5 \mathrm{mg} \mathrm{mL}^{-1}\right.$ DNase, $0.25 \mathrm{mg} \mathrm{mL}^{-1}$ RNase, $50 \mathrm{mmol} \mathrm{L}^{-1} \mathrm{MgCl}_{2}$ ). Protein was precipitated with $30 \%$ TCA. Finally, the protein pellets were air-dried and dissolved in a solubilization buffer containing $7 \mathrm{~mol} \mathrm{~L}^{-1}$ urea, $2 \mathrm{~mol} \mathrm{~L}^{-1}$ thiourea, $4 \%(\mathrm{w} / \mathrm{v})$ CHAPS, $1 \%(\mathrm{w} / \mathrm{v})$ 
dithiothreitol (DTT), $20 \mathrm{mmol} \mathrm{L}^{-1}$ Tris, and 1\% (v/v) Pharmalyte $^{\mathrm{TM}} \mathrm{pH}$ 3-10 (Amersham Biosciences). The total soluble protein concentration was determined using the BIO-RAD protein assay (BIO-RAD Lab., Hartfordshire, USA). The solubilized proteins were stored at $-70^{\circ} \mathrm{C}$ until further analysis.

\section{Two-dimensional gel electrophoresis}

Two-dimensional (2-D) gel electrophoresis was essentially carried out as described previously [17]. Briefly, the first-dimension using isoelectric focusing (IEF) was run with the IPGphor ${ }^{\mathrm{TM}}$ Isoelectric Focusing System (Amersham Biosciences) loading $300 \mu \mathrm{g}$ protein sample onto Immobiline DryStrip gels of pH 3-10 (IPG strips, Amersham Biosciences) by in-gel rehydration. IEF was performed with the following setting: $30 \mathrm{~V} \times 12 \mathrm{~h}$, $300 \mathrm{~V} \times 3 \mathrm{~h}, 600 \mathrm{~V} \times 2 \mathrm{~h}, 1000 \mathrm{~V} \times 1 \mathrm{~h}$, gradient from $1000 \mathrm{~V}$ to $5000 \mathrm{~V}$ within $2 \mathrm{~h}, 5000 \mathrm{~V} \times 2 \mathrm{~h}$, gradient from $5000 \mathrm{~V}$ to $8000 \mathrm{~V}$ within $2 \mathrm{~h}$, then $8000 \mathrm{~V} \times 10 \mathrm{~h}$. Prior to the second dimension (SDS-PAGE), the IPG strips were equilibrated and then transferred onto lab cast SDS-polyacrylamide gels (DALT multiple gel caster and DALT gradient maker, Amersham Biosciences). Proteins were separated on $12-16 \%$ linear gradient gels using the vertical separation unit Hoefer $^{\text {тM }}$ System (Amersham Biosciences). Subsequently, gels were stained using colloidal Coomassie Blue G-250 according to the "Blue silver" protocol [47]. The gels were then scanned (ScanMaker 9800 XL, Umax System GmbH, Germany) at 300 dpi resolution. Image analysis, namely protein spot detection, matching and quantification were performed using Proteomweaver ${ }^{\mathrm{TM}}$ 3.0 (Definens AG, Germany).

\section{In-gel trypsin digestion and peptide extraction}

The intracellular proteome was analyzed at different cultivation time points by 2-D gel electrophoresis combined with Maldi-ToF analysis. A total of 136 protein spots were excised manually from Coomassie brilliant Blue stained 2-D gels, each spot was washed several times with $200 \mu \mathrm{l}$ water, dehydrated in $50 \mu \mathrm{l}$ acetonitrile, and dried in a vacuum concentrator (Eppendorf ${ }^{\circledR}$ Vacufuge Concentrator 5301, Eppendorf AG, Hamburg). The gel pieces were treated with $100 \mathrm{mmol} \mathrm{L}^{-1}$ ammonium bicarbonate containing $20 \mathrm{mmol} \mathrm{L}^{-1} \mathrm{DTT}$ at $56^{\circ} \mathrm{C}$ for $30 \mathrm{~min}$ and then with $100 \mathrm{mmol} \mathrm{L}^{-1}$ ammonium bicarbonate containing $55 \mathrm{mmol} \mathrm{L}^{-1}$ iodoacetamide in the dark at room temperature for $30 \mathrm{~min}$. Acetonitrile was added in between the treatments to dehydrate the gel pieces. Finally, the gel pieces were washed twice with $100 \mathrm{mmol} \mathrm{L}^{-1}$ ammonium bicarbonate, dehydrated with acetonitrile and dried in the vacuum concentrator. Ingel digestion was carried out by incubation with $2 \mathrm{ng}$ $\mu \mathrm{L}^{-1}$ trypsin (sequencing grade modified, Promega
Corp.) in $50 \mathrm{mmol} \mathrm{L}^{-1}$ ammonium bicarbonate at $37^{\circ} \mathrm{C}$ overnight. Obtained peptides were extracted and then desalted with reversed-phased C-18 ZipTips (Millipore, Billerica, MA, USA) before application to the Maldi-ToF sample plates.

\section{Maldi-ToF MS analysis}

Matrix-assisted laser desorption ionization time-of-flight mass spectrometry (Maldi-ToF MS) was employed to obtain the peptide mass fingerprint of a given protein. The concentrated peptide solution was mixed $(1: 1, \mathrm{v} / \mathrm{v})$ with the MALDI loading solution (10 mg $\alpha$-cyano-4hydoxycinnamic acid, $400 \mu \mathrm{L}$ acetonitrile and $600 \mu \mathrm{L}$ $0.1 \%$ trifluoroacetic acid), loaded on the target and dried at room temperature. The molecular masses of the tryptic peptides were determined on a Bruker Ultraflex time-of-flight mass spectrometer (Bruker Daltonics $\mathrm{GmbH}$, Germany).

\section{Data analysis}

Peptide mass fingerprints obtained by the Maldi-ToF MS were processed using FlexAnalysis 2.0 (Bruker Daltonik GmbH, Germany) and used to search NCBInr database by using Mascot 2.10 software (http://www. matrixscience.com). The parameters used for the search were as follows: taxonomy: other Fungi, tryptic digestion, modifications were allowed for carbamidomethylation of cysteine (fixed modification) and methionine oxidation (variable modification), one missed cleavage site was allowed, all peptides monoisotopic, peptide tolerance at $100 \mathrm{ppm}$. Mascot scores (probability based MOWSE scores) and expect values were generated from the Mascot search program. All proteins with a Mowse score $\geq 70$ were regarded as significant $(p<0.05)$. Protein identification was based on the recently annotated P. pastoris GS115 genome sequence [48] and the gene name used in this study is according to Pichia pastoris strain GS115 (http://www.uniprot.org/). If no gene name was given for this strain the gene name is according to Pichia pastoris strain ATCC 76273 / CBS 7435 / CECT 11047 / NRRL Y-11430 / Wegner 21-1 or general Pichia pastoris (yeast) in case of $100 \%$ sequence identity (http://www.uniprot.org/). Image analysis from the scanned gels, namely protein spot detection, matching and quantification were performed using Proteomwea$\operatorname{ver}^{\mathrm{TM}} 3.0$ (Definiens AG, Germany). The spot volumes were computed and normalized for each spot on each gel in relation to the total spot volume of each $2 \mathrm{D}$ gel. To obtain comparable data, spot intensities were normalized using the log2 ratio of induced samples versus uninduced sample. Log2 fold changes above 0.6 (equivalent to a 1.5 fold changes) were considered significant. 


\section{Ethanol analysis}

Ethanol concentrations were determined by gas chromatography (Shimadzu 14B GC, Kyoto, Japan) using a column packed with Carbograph 1AW (20/120; 5\% carbowax $20 \mathrm{M}$ ) (Alltech Associates Inc., Deerfield, IL, USA), column temperature $160^{\circ} \mathrm{C}$, detector: flame ionization detector.

\section{Electron microscopy and image analysis}

Electron microscopic studies were carried out essentially as described previously [14]. Mitochondrial and total cell area were determined from randomly chosen cells at the end of the glycerol batch phase (in total: 55 mitochondria in 12 cells) and after 112 hours of growth on methanol (in total: 67 mitochondria in 8 cells) using Image J Software (National Institutes of Health, Bethesda, Maryland, USA). For analysis of vacuolar morphology, 520 cells were randomly chosen for analysis (224 cells at the end of the glycerol batch phase, 160 cells 48 hours and 136 cells 112 hours after the onset of methanol feeding).

\section{Additional files}

Additional file 1: All identified intracellular proteins. The complete list of all identified proteins classified into functional categories and their log2 changes in response to methanol-induced high-level production of the $\mathrm{HBsAg}$ and a representative $2 \mathrm{D}$ gel image indicating the spots of all identified proteins are given.

Additional file 2: Identification of AOX1 in P. pastoris GS115 with a "Mut ${ }^{\mathrm{s}}$ phenotype". Unexpectedly AOX1 was identified in the supposedly Mut' strain of P. pastoris GS115 producing high levels of HBsAg. Background information on AOX1 identification and discussion about Mut ${ }^{5}$ behavior of Mut $^{+}$strain is given.

\section{Competing interests}

The authors declare that they have no competing interests.

\section{Authors' contributions}

AV identified the proteins by Maldi-ToF, analyzed the data and prepared a first draft of the manuscript. $H L$ prepared the electron micrographs. AA and CG carried out the cultivation. AA also prepared the 2D gels. MN contributed to protein identification by Maldi-ToF. NK was involved in the initial outline of the project. UR conceived and directed the study and prepared the final manuscript. All authors read and approved the final manuscript.

\section{Acknowledgements}

Ana Letícia Vanz would like to acknowledge the Federal Agency for the Improvement of Higher Education, Brazil (CAPES) for providing a PhD fellowship. Ahmad Adnan wishes to express his gratitude to the Higher Education Commission (HEC) of Pakistan for a post-doctoral fellowship. Partial support through an Indo-German program funded by DBT (India) and BMBF (Germany) is also gratefully acknowledged. We are also grateful to Ingeborg Kristen and Anja Meier for skilful support in TEM and MS sample preparations (VAM and CPRO, HZI). Moreover, we thank Frank Stahl for helpful support and discussions concerning primer design and PCR analysis.

\section{Author details}

${ }^{1}$ Technical Chemistry - Life Science, Leibniz University of Hannover, Hannover, Germany. ${ }^{2}$ Helmholtz Centre for Infection Research, Inhoffenstrasse 7, Braunschweig 38124, Germany. ${ }^{3}$ Department of Chemistry, Government College University Lahore, Lahore, Pakistan. ${ }^{4}$ International Centre for Genetic Engineering \& Biotechnology, New Delhi, India.
Received: 31 May 2012 Accepted: 28 July 2012

Published: 8 August 2012

\section{References}

1. Mattanovich D, Branduardi P, Dato L, Gasser B, Sauer M, Porro D: Recombinant protein production in yeasts. Methods Mol Biol 2012, 824:329-358.

2. Cereghino GP, Cereghino JL, Ilgen C, Cregg JM: Production of recombinant proteins in fermenter cultures of the yeast Pichia pastoris. Curr Opin Biotechnol 2002, 13:329-332.

3. Cregg JM, Madden KR, Barringer KJ, Thill GP, Stillman CA: Functional characterization of the two alcohol oxidase genes from the yeast Pichia pastoris. Mol Cell Biol 1989, 9:1316-1323.

4. Vassileva A, Chugh DA, Swaminathan S, Khanna N: Effect of copy number on the expression levels of hepatitis $B$ surface antigen in the methylotrophic yeast Pichia pastoris. Protein Expr Purif 2001, 21:71-80.

5. Zhu T, Guo M, Tang Z, Zhang M, Zhuang Y, Chu J, et al: Efficient generation of multi-copy strains for optimizing secretory expression of porcine insulin precursor in yeast Pichia pastoris. J Appl Microbiol 2009, 107:954-963.

6. Clare JJ, Romanos MA, Rayment FB, Rowedder JE, Smith MA, Payne MM, et al: Production of mouse epidermal growth factor in yeast: high-level secretion using Pichia pastoris strains containing multiple gene copies. Gene 1991, 105:205-212.

7. Mansur M, Cabello C, Hernandez L, Pais J, Varas L, Valdes J, et al: Multiple gene copy number enhances insulin precursor secretion in the yeast Pichia pastoris. Biotechnol Lett 2005, 27:339-345.

8. Zhu T, Guo M, Zhuang Y, Chu J, Zhang S: Understanding the effect of foreign gene dosage on the physiology of Pichia pastoris by transcriptional analysis of key genes. App/ Microbiol Biotechnol 2011, 89:1127-1135.

9. Hohenblum H, Gasser B, Maurer M, Borth N, Mattanovich D: Effects of gene dosage, promoters, and substrates on unfolded protein stress of recombinant Pichia pastoris. Biotechnol Bioeng 2004, 85:367-375.

10. Cos O, Serrano A, Montesinos JL, Ferrer P, Cregg JM, Valero F: Combined effect of the methanol utilization (Mut) phenotype and gene dosage on recombinant protein production in Pichia pastoris fed-batch cultures. J Biotechnol 2005, 116:321-335.

11. Gasser B, Saloheimo M, Rinas U, Dragosits M, Rodríguez-Carmora E, Baumann $\mathrm{K}$, et al: Conformational stress in microbial cells producing recombinant proteins: a host comparative overview. Microb Cell Fact 2008, 7:11

12. Whyteside G, Alcocer MJC, Kumita JR, Dobson CM, Lazarou M, Pleass RJ, et al: Native-state stability determines the extent of degradation relative to secretion of protein variants from Pichia pastoris. PLOS One 2011, 6:e22692.

13. Gasser B, Maurer M, Rautio J, Sauer M, Bhattacharyya A, Saloheimo M, et al: Monitoring of transcriptional regulation in Pichia pastoris under protein production conditions. BMC Genomics 2007, 8:179.

14. Lünsdorf H, Gurramkonda C, Adnan A, Khanna N, Rinas U: Virus-like particle production with yeast: ultrastructural and immunocytochemical insights into Pichia pastoris producing high levels of the Hepatitis B surface antigen. Microb Cell Fact 2011, 10:48.

15. Gurramkonda C, Adnan A, Gäbel T, Lünsdorf H, Ross A, Nemani SK, et al: Simple high-cell density fed-batch technique for high-level recombinant protein production with Pichia pastoris: Application to intracellular production of Hepatitis B surface antigen. Microb Cell Fact 2009, 8:13.

16. Hartner FS, Glieder A: Regulation of methanol utilisation pathway genes in yeasts. Microb Cell Fact 2006, 5:39.

17. Lu X, Sun J, Nimtz M, Wissing J, Zeng AP, Rinas U: The intra- and extracellular proteome of Aspergillus niger growing on defined medium with xylose or maltose as carbon substrate. Microb Cell Fact 2010, 9:23.

18. Inan M, Meagher MM: The effect of ethanol and acetate on protein expression in Pichia pastoris. J Biosci Bioeng 2001, 92:337-341.

19. Chiruvolu V, Eskridge K, Cregg J, Meagher M: Effects of glycerol concentration and $\mathrm{pH}$ on growth of recombinant Pichia pastoris yeast. Appl Biochem Biotechnol 1998, 75:163-173.

20. Yurimoto H, Lee B, Yasuda F, Sakai Y, Kato N: Alcohol dehydrogenases that catalyse methyl formate synthesis participate in formaldehyde detoxification in the methylotrophic yeast Candida boidinii. Yeast 2004, 21:341-350. 
21. Horiguchi H, Yurimoto H, Goh T, Nakagawa T, Kato N, Sakai Y: Peroxisomal catalase in the methylotrophic yeast Candida boidinii: transport efficiency and metabolic significance. J Bacteriol 2001, 183:6372-6383.

22. Bener Aksam E, de Vries B, van der Klei IJ, Kiel JAKW: Preserving organelle vitality: peroxisomal quality control mechanisms in yeast. FEMS Yeast Res 2009, 9:808-820.

23. Horiguchi $H$, Yurimoto $H$, Kato N, Sakai $Y$ : Antioxidant system within yeast peroxisome. Biochemical and physiological characterization of CbPmp20 in the methylotrophic yeast Candida boidinii. J Biol Chem 2001, 276:14279-14288.

24. Yano T, Takigami E, Yurimoto H, Sakai Y: Yap1-regulated glutathione redox system curtails accumulation of formaldehyde and reactive oxygen species in methanol metabolism of Pichia pastoris. Eukaryot Cell 2009, 8:540-549.

25. Bener Aksam E, Jungwirth $H$, Kohlwein SD, Ring J, Madeo F, Veenhuis M, et al: Absence of the peroxiredoxin Pmp20 causes peroxisomal protein leakage and necrotic cell death. Free Radic Biol Med 2008, 45:1115-1124.

26. Kim IS, Yun HS, Park IS, Sohn HY, Iwahashi H, Jin IN: A knockout strain of CPR1 induced during fermentation of Saccharomyces cerevisiae KNU5377 is susceptible to various types of stress. J Biosci Bioeng 2006, 102:288-296.

27. Zhao Q, Wang Y, Freed D, Fu TM, Gimenez JA, Sitrin RD, et al: Maturation of recombinant hepatitis $B$ virus surface antigen particles. Hum Vaccin 2006, 2:174-180

28. Graf A, Gasser B, Dragosits M, Sauer M, Leparc GG, Tuchler T, et al: Novel insights into the unfolded protein response using Pichia pastoris specific DNA microarrays. BMC Genomics 2008, 9:390.

29. Taxis C, Hitt R, Park SH, Deak PM, Kostova Z, Wolf DH: Use of modular substrates demonstrates mechanistic diversity and reveals differences in chaperone requirement of ERAD. J Biol Chem 2003, 278:35903-35913.

30. Hoseki J, Ushioda R, Nagata K: Mechanism and components of endoplasmic reticulum-associated degradation. J Biochem 2010, 147:19-25.

31. Zolkiewski M, Zhang T, Nagy M: Aggregate reactivation mediated by the Hsp100 chaperones. Arch Biochem Biophys 2012, 520: 1-6.

32. Glover JR, Lindquist S: Hsp104, Hsp70, and Hsp40: a novel chaperone system that rescues previously aggregated proteins. Cell 1998, 94:73-82

33. Rabinovich E, Kerem A, Frohlich KU, Diamant N, Bar-Nun S: AAA-ATPase p97/Cdc48p, a cytosolic chaperone required for endoplasmic reticulum-associated protein degradation. Mol Cell Biol 2002, 22:626-634

34. Stolz A, Hilt W, Buchberger A, Wolf DH: Cdc48: a power machine in protein degradation. Trends Biochem Sci 2011, 36:515-523.

35. Wolf $\mathrm{DH}$, Stolz $\mathrm{A}$ : The $\mathrm{Cdc} 48$ machine in endoplasmic reticulum associated protein degradation. Biochim Biophys Acta 2012, 1823:117-124.

36. Baba M, Takeshige K, Baba N, Ohsumi Y: Ultrastructural analysis of the autophagic process in yeast: detection of autophagosomes and their characterization. J Cell Biol 1994, 124:903-913.

37. Dunn WA Jr, Cregg JM, Kiel JAKW, van der Klei I, Oku M, Sakai Y, et al: Pexophagy: the selective autophagy of peroxisomes. Autophagy 2005, 1:75-83.

38. Sakai Y, Koller A, Rangell LK, Keller GA, Subramani S: Peroxisome degradation by microautophagy in Pichia pastoris: identification of specific steps and morphological intermediates. J Cell Biol 1998, 141: 625-636.

39. Ano Y, Hattori T, Kato N, Sakai Y: Intracellular ATP correlates with mode of pexophagy in Pichia pastoris. Biosci Biotechnol Biochem 2005, 69:1527-1533

40. Bernales S, McDonald KL, Walter P: Autophagy counterbalances endoplasmic reticulum expansion during the unfolded protein response. PLoS Biol 2006, 4:e423.

41. Ogata M, Hino S, Saito A, Morikawa K, Kondo S, Kanemoto S, et al: Autophagy is activated for cell survival after endoplasmic reticulum stress. Mol Cell Biol 2006, 26:9220-9231.

42. Pfeffer M, Maurer M, Stadlmann J, Grass J, Delic M, Altmann F, et al: Intracellular interactome of secreted antibody Fab fragment in Pichia pastoris reveals its routes of secretion and degradation. Appl Microbiol Biotechnol 2012, 93:2503-2512.

43. Aksam EB, Koek A, Kiel JAKW, Jourdan S, Veenhuis M, van der Klei IJ: A peroxisomal lon protease and peroxisome degradation by autophagy play key roles in vitality of Hansenula polymorpha cells. Autophagy 2007, 3:96-105.
44. van Zutphen $\mathrm{T}$, Veenhuis $\mathrm{M}$, van der Klei IJ: Damaged peroxisomes are subject to rapid autophagic degradation in the yeast Hansenula polymorpha. Autophagy 2011, 7:863-872.

45. Krainer FW, Dietzsch C, Hajek T, Herwig C, Spadiut O, Glieder A Recombinant protein expression in Pichia pastoris strains with an engineered methanol utilization pathway. Microb Cell Fact 2012, 11:22.

46. Kimura S, Maruyama J, Kikuma T, Arioka M, Kitamoto K: Autophagy delivers misfolded secretory proteins accumulated in endoplasmic reticulum to vacuoles in the filamentous fungus Aspergillus oryzae. Biochem Biophys Res Commun 2011, 406:464-470.

47. Candiano G, Bruschi M, Musante L, Santucci L, Ghiggeri GM, Carnemolla B, et al: Blue silver: a very sensitive colloidal Coomassie G-250 staining for proteome analysis. Electrophoresis 2004, 25:1327-1333.

48. De Schutter K, Lin YC, Tiels P, Van HA, Glinka S, Weber-Lehmann J, et al: Genome sequence of the recombinant protein production host Pichia pastoris. Nat Biotechnol 2009, 27:561-566.

\section{doi:10.1186/1475-2859-11-103}

Cite this article as: Vanz et al: Physiological response of Pichia pastoris GS115 to methanol-induced high level production of the Hepatitis B surface antigen: catabolic adaptation, stress responses, and autophagic processes. Microbial Cell Factories 2012 11:103.

\section{Submit your next manuscript to BioMed Central and take full advantage of:}

- Convenient online submission

- Thorough peer review

- No space constraints or color figure charges

- Immediate publication on acceptance

- Inclusion in PubMed, CAS, Scopus and Google Scholar

- Research which is freely available for redistribution 